\title{
Semialgebraic version of Whitney's extension theorem
}

\author{
Beata Kocel-Cynk, WiesŁaw Pawłucki(D, and Anna Valette
}

\begin{abstract}
In this note we prove a semialgebraic counterpart of Whitney's extension theorem.
\end{abstract}

Mathematics Subject Classification. 58C25, 14P20, 57R35, 03C64.

Keywords. Whitney field, Extension theorem, $\mathcal{C}^{p}$-functions, Nash functions.

Semialgebraic geometry plays an important role in areas of pure mathematics such as singularity theory and mathematical logic. It appears also very valuable in areas of applied mathematics such as robotics and CAD. Together with its generalization (subanalytic geometry, o-minimal geometry) it serves as a tool for problems in analysis [4,7].

It is therefore a very natural question if the famous Whitney extension theorem admits a semialgebraic counterpart. Let us first recall this fundamental result of analysis.

For a subset $A$ of $\mathbb{R}^{n}$, denote by $\mathcal{C}(A)$ the algebra of real continuous functions on $A$.

Definition 1. Let $p$ be a positive integer. A $\mathcal{C}^{p}-$ Whitney field on the set $A$ is a polynomial

$$
F(u, X)=\sum_{|\kappa| \leq p} \frac{1}{\kappa !} F^{\kappa}(u) X^{\kappa} \in \mathcal{C}(A)[X]=\mathcal{C}(A)\left[X_{1}, \ldots, X_{n}\right]
$$

which fulfills the following condition: for each $c \in A$ and every $\alpha \in \mathbb{N}^{n}$ such that $|\alpha| \leq p$,

$$
D_{X}^{\alpha} F(a, 0)-D_{X}^{\alpha} F(b, a-b)=o\left(|a-b|^{p-|\alpha|}\right),
$$

when $A \times A \ni(a, b) \rightarrow(c, c)$. 
Whenever $A$ is open in $\mathbb{R}^{n}$, any $\mathcal{C}^{p}$-Whitney field can be identified with a $\mathcal{C}^{p}$-function on $A$.

Whitney's extension theorem (cf. [11]) asserts that given a closed subset $A$ of $\mathbb{R}^{n}$ and any $\mathcal{C}^{p}$-Whitney field $F$ on $A$, there exists a $\mathcal{C}^{p}$-function $f: \mathbb{R}^{n} \rightarrow \mathbb{R}$ such that $D^{\kappa} f=F^{\kappa}$ on $A$, if $\kappa \in \mathbb{N}^{n},|\kappa| \leq p$, and the restriction $f_{\mid \mathbb{R}^{n} \backslash A}$ : $\mathbb{R}^{n} \backslash A \rightarrow \mathbb{R}$ is analytic.

We refer the reader to [1] or [10] for basic definitions and properties of semialgebraic sets.

In this note we establish the semialgebraic counterpart of this theorem. Firstly, let us remark that using the so-called $\Lambda_{p}$-regular stratifications, the second author and K. Kurdyka proved an o-minimal analogue of Whitney's extension theorem, containing in particular the following semialgebraic version (cf. [5] and [6, Theorem 1]).

Theorem 2. Let $E$ be a semialgebraic closed subset of an open semialgebraic subset $\Omega$ of $R^{n}$, and let $p$ and $q$ be positive integers such that $p \leq q$. Let

$$
F(x, X)=\sum_{|\kappa| \leq p} \frac{1}{\kappa !} F^{\kappa}(x) X^{\kappa}
$$

be a semialgebraic $\mathcal{C}^{p}$ - Whitney field on $E$ (i.e. all $F^{\kappa}$ are semialgebraic functions). Then there exists a semialgebraic $\mathcal{C}^{p}$-function $f: \Omega \rightarrow \mathbb{R}, \mathcal{C}^{q}$ on $\Omega \backslash E$, such that $D^{\kappa} f=F^{\kappa}$ on $E$ whenever $\kappa \in \mathbb{N}^{n}$ with $|\kappa| \leq p$.

We want to use Shiota's approximation theorem to get the function $f$ Nash on $\Omega \backslash E$. Recall that a semialgebraic subset $M$ of $\mathbb{R}^{n}$ is called a Nash submanifold if it is an analytic submanifold of $\mathbb{R}^{n}$ and a map $f: M \rightarrow N$ of Nash submanifolds is called a Nash map if it is an analytic map with semialgebraic graph. Actually, in the semialgebraic case the analyticity is equivalent to $\mathcal{C}^{\infty}$ (cf. [8, Proposition 3.11]). It is well known that every Nash submanifold admits a finite system of Nash charts (cf. [10]).

Let $p$ be a positive integer. We briefly recall the definition of the semialgebraic $\mathcal{C}^{p}$-topology. Let $M \subset \mathbb{R}^{n}$ and $N \subset \mathbb{R}^{m}$ be Nash submanifolds of dimension $k$ and $l$, respectively. Denote by $\mathcal{N}^{p}(M, N)$ the set of $\mathcal{C}^{p}$ semialgebraic maps from $M$ to $N$, i.e. $\mathcal{C}^{p}$ maps with semialgebraic graphs.

Fix any $f \in \mathcal{N}^{p}(M, N)$. Then there exist finite systems $\Phi=\left\{\varphi_{i}, U_{i}\right\}_{i \in S}$ (resp. $\Psi=\left\{\psi_{i}, V_{i}\right\}_{i \in S}$ ) of Nash charts on $M$ (resp. on $N$ ) and a family of closed sets $\left\{Z_{i}\right\}_{i \in S}$ of $M$ such that $\bigcup_{i \in S} Z_{i}=M$ and for each $i \in S, Z_{i} \subset U_{i}$, and $f\left(Z_{i}\right) \subset V_{i}$. Now basic neighborhoods of $f$ in the semialgebraic $\mathcal{C}^{p}$-topology in $\mathcal{N}^{p}(M, N)$ are of the form

$$
\begin{aligned}
U_{\varepsilon}(f)= & \left\{g \in \mathcal{N}^{p}(M, N): \text { for each } i \in S, \lambda \in \mathbb{N}^{k},|\lambda| \leq p, \xi \in \varphi_{i}\left(Z_{i}\right), g\left(Z_{i}\right) \subset V_{i},\right. \\
& \left.\left|D^{\lambda}\left(\psi_{i} f \varphi_{i}^{-1}\right)(\xi)-D^{\lambda}\left(\psi_{i} g \varphi_{i}^{-1}\right)(\xi)\right| \leq \varepsilon\left(\varphi_{i}^{-1}(\xi)\right)\right\},
\end{aligned}
$$

where $\varepsilon: M \rightarrow(0,+\infty)$ is any semialgebraic positive continuous function on $M$.

Clearly, if $M=\Omega$ is an open semialgebraic subset of $\mathbb{R}^{n}$ and $N=\mathbb{R}$, then

$$
\begin{aligned}
U_{\varepsilon}(f)= & \left\{g \in \mathcal{N}^{p}(\Omega, \mathbb{R}):\left|D^{\lambda} f(x)-D^{\lambda} g(x)\right| \leq \varepsilon(x)\right. \\
& \text { whenever } \left.\lambda \in \mathbb{N}^{n},|\lambda| \leq p, x \in \Omega\right\} .
\end{aligned}
$$


Theorem 3. (Shiota's approximation theorem, $[9,10])$. Let $M \subset \mathbb{R}^{n}$ and $N \subset$ $\mathbb{R}^{m}$ be Nash submanifolds and $f: M \rightarrow N$ a semialgebraic $\mathcal{C}^{p}$ map. Then $f$ can be approximated by Nash maps in the semialgebraic $\mathcal{C}^{p}$-topology.

Now the semialgebraic Whitney extension theorem can be formulated as follows.

Theorem 4. Let $E$ be a semialgebraic closed subset of an open semialgebraic subset $\Omega$ of $\mathbb{R}^{n}, p \in \mathbb{N}$, and let

$$
F(x, X)=\sum_{|\kappa|=0}^{p} \frac{1}{\kappa !} F^{\kappa}(x) X^{\kappa}
$$

be a semialgebraic $\mathcal{C}^{p}$ Whitney field on $E$. Then there exists a $\mathcal{C}^{p}$ semialgebraic function $f: \Omega \rightarrow \mathbb{R}$ such that $D^{\kappa} f=F^{\kappa}$ on $E$ for $|\kappa| \leq p$ and $f$ is Nash on the set $\Omega \backslash E$.

Proof. Thanks to Theorem 2, there exists a semialgebraic $\mathcal{C}^{p}$ function $\tilde{f}: \Omega \rightarrow$ $\mathbb{R}$ such that on the set $E$ the equality $D^{\kappa} \tilde{f}=F^{\kappa}$ holds for any $|\kappa| \leq p$. Applying Shiota's approximation theorem to the function $\tilde{f}$, and $\varepsilon(x):=\operatorname{dist}(x, E)$ we get a function $f: \Omega \rightarrow \mathbb{R}$ such that $f$ is Nash on the set $\Omega \backslash E$ and

$$
\left|D^{\kappa} f(x)-D^{\kappa} \tilde{f}(x)\right|<\varepsilon(x)
$$

for every $x \in \Omega \backslash E$ and any $|\kappa| \leq p$. Hence $D^{\kappa} \tilde{f}=D^{\kappa} f=F^{\kappa}$ for all $|\kappa| \leq p$ on the set $E$ as required.

Remark 5. A counterpart of this theorem is also valid for non polynomially bounded o-minimal structures and more generally on every o-minimal structure on which Shiota's approximation theorem holds true ([3]). It is however unclear whether Theorem 4 is true for all polynomially bounded o-minimal structures as it is still unknown whether Shiota's theorem holds in this case. In particular, we do not know whether it is possible to generalize the Whitney extension theorem to the globally subanalytic category which is an interesting open problem.

Open Access. This article is distributed under the terms of the Creative Commons Attribution 4.0 International License (http://creativecommons.org/licenses/ by $/ 4.0 /$ ), which permits unrestricted use, distribution, and reproduction in any medium, provided you give appropriate credit to the original author(s) and the source, provide a link to the Creative Commons license, and indicate if changes were made.

Publisher's Note Springer Nature remains neutral with regard to jurisdictional claims in published maps and institutional affiliations.

\section{References}

[1] Bochnak, J., Coste, M., Roy, M.-F.: Géométrie algébrique réelle. Springer, Berlin (1987) 
[2] Efroymson, G.A.: The Extension Theorem for Nash Functions. Real algebraic geometry and quadratic forms (Rennes, 1981), 343-357, Lecture Notes in Math. 959, Springer, Berlin (1982)

[3] Fischer, A.: Smooth functions in o-minimal structures. Adv. Math. 218, 496-514 (2008)

[4] Hörmander, L.: On the division of distributions by polynomials. Ark. Mat. 3, 555-568 (1958)

[5] Kurdyka, K., Pawłucki, W.: Subanalytic version of Whitney's extension theorem. Studia Math. 124, 269-280 (1997)

[6] Kurdyka, K., Pawłucki, W.: O-minimal version of Whitney's extension theorem. Studia Math. 224(1), 81-96 (2014)

[7] Łojasiewicz, S.: Sur le problème de la division (French). Studia Math. 18, 87-136 (1959)

[8] Malgrange, B.: Ideals of Differentiable Functions. Oxford University Press, Oxford (1966)

[9] Shiota, M.: Approximation theorems for Nash mappings and Nash manifolds. Trans. Am. Math. Soc. 293(1), 319-337 (1986)

[10] Shiota, M.: Nash Manifolds. Lecture Notes in Mathematics, vol. 1269. Springer, Berlin (1987)

[11] Whitney, H.: Analytic extensions of differentiable functions defined in closed sets. Trans. Am. Math. Soc. 36(1), 63-89 (1934)

BeATA KOCEL-CYNK

Instytut Matematyki Politechniki Krakowskiej

ul. Warszawska 24

31-155 Kraków

Poland

e-mail: bkocel@pk.edu.pl

Wieseaw PaWŁucki And Anna Valette

Instytut Matematyki Uniwersytetu Jagiellońskiego

ul. S. Łojasiewicza 6

30-348 Kraków

Poland

e-mail: wieslaw.pawlucki@im.uj.edu.pl

Anna Valette

e-mail: anna.valette@im.uj.edu.pl

Received: 14 November 2018 